\title{
Disfunção tiróidea e amiodarona
}

\author{
Thyroid dysfunction and amiodarone
}

${ }^{1}$ Serviço de Medicina Interna dos Hospitais de Universidade de Coimbra e Faculdade de Medicina da Universidade de Coimbra, Coimbra, Portugal

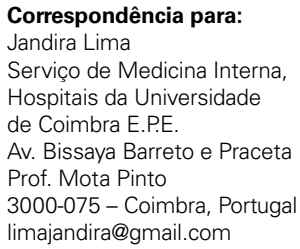

Recebido em 25/Jan/2012 Aceito em 21/Jun/2012
Jandira Lima', Patrícia Carvalho', M. Auxiliadora Molina', Marta Rebelo', Patrícia Dias', José Diniz Vieira', José M. Nascimento Costa'

\begin{abstract}
SUMÁRIO
Apesar de a maioria dos doentes tratados com amiodarona permanecer em eutiroidia, alguns desenvolvem hipertiroidismo (HPEIA) ou hipotiroidismo (HPOIA) induzidos pela amiodarona. Os autores apresentam uma análise retrospectiva dos processos de dez doentes com disfunção tiróidea induzida pela amiodarona. Verificou-se que seis doentes eram mulheres e que o tempo médio de toma da amiodarona foi de 17,7 meses. O HPOIA foi o mais frequente (seis doentes). Dos doentes com HPEIA, dois tinham HPEIA tipo 2, um tipo 1 e um tipo 3. Sintomas sugestivos de disfunção tiróidea ocorreram em cinco doentes, a maioria com HPOIA. No HPEIA, a clínica mais comum foi exacerbação da arritmia de base (três doentes). $A$ interrupção da amiodarona e administração de levotiroxina foi a terapêutica escolhida em $83,3 \%$ dos casos de HPOIA, enquanto a tionamida associada a corticoide com suspensão da amiodarona foi opção em $75 \%$ dos casos de HPEIA. Registraram-se três óbitos, todos com HPEIA. O HPEIA constituiu uma complicação potencialmente fatal. A clínica pode ser vaga, pelo que a monitorização da função tiróidea é obrigatória. Arq Bras Endocrinol Metab. 2013;57(1):71-8
\end{abstract}

\section{SUMMARY}

Although most patients remain clinically euthyroid, some develop amiodarone-induced hyperthyroidism (HPEAl) or hypothyroidism (HPOAI). The authors present a retrospective analysis of ten patients with amiodarone-induced thyroid dysfunction. Six patients were female and mean amiodarone intake was 17.7 months. HPOIA was more common (six patients). From all the patients with HPEAl, two had type 2, one had type 1, and one had type 3 hyperthyroidism. Symptoms suggestive of thyroid dysfunction occurred in five patients, most of them with HPOAI. In HPEAl, the most frequent symptom was exacerbation of arrhythmia (three patients). Discontinuation of amiodarone and treatment with levothyroxine was chosen in $83.3 \%$ of the HPOAI cases, while thyonamide treatment with corticosteroids and without amiodarone was the option in $75 \%$ of the HPEAl cases. There were three deaths, all in patients with HPEAI. HPEAI is potentially fatal. The clinical picture may be vague, so the thyroid monitoring is mandatory. Arq Bras Endocrinol Metab. 2013;57(1):71-8

\section{INTRODUÇÃO}

A disfunção tiróidea induzida pela amiodarona (DTIA) é uma complicação potencialmente grave, cuja incidência é estimada entre $2 \%$ e $24 \%(1,2)$.

A amiodarona, um antiarrítmico da classe 3 da classificação de Vaughan Williams, introduzida em 1962 como antianginoso, foi aprovada pela Food and Drug Administration em 1985 para o tratamento de taquiarritmias supraventriculares $(1,2)$. Constitui um dos fármacos mais utilizados atualmente para manutenção do ritmo sinusal em doentes com fibrilhação auricular (1) e recentemente foi incluída nas diretivas da American Heart Association como um dos fármacos utilizados na paragem cardíaca (3). Contudo, está associada a vários efeitos secundários, que ocorrem em cerca de $80 \%$ dos doentes. Os efeitos mais frequentes são microdepósitos córneos (quase 100\%), perturbações gastrointestinais benignas $(80 \%)$, fotossensibilidade e hiperpigmentação cutânea nas áreas expostas (55\% a 75\%), e sintomas neurológicos como tremor, ataxia e neuropatia periférica sensitivomotora $(48 \%)$. Os efeitos mais graves, mas menos frequentes, são falência hepática, toxicidade pulmonar e pró-arritmias (4).

É um derivado benzofurânico, estruturalmente semelhante à tiroxina (T4) e à triiodotironina (T3), que contém dois átomos de iodo por molécula (equivalente 
a $37,5 \%$ do seu peso) (5). Assim, cada dose de $200 \mathrm{mg}$ de amiodarona contém $75 \mathrm{mg}$ de iodo, dos quais se estipulam ser libertados $6 \mathrm{mg}$ como iodo livre diariamente, excedendo a dose dietética diária recomendada pela World Health Organization (WHO) $(0,2$ a 0,8 $\mathrm{mg} /$ dia $)$ em 20 a 40 vezes $(2,5-7)$. Sua biodisponibilidade oral varia entre $30 \%$ e $80 \%$ e possui um grande volume de distribuição, em decorrência da extensa acumulação no tecido adiposo $(316 \mu \mathrm{g} / \mathrm{g})$, fígado (391 $\mu \mathrm{g} / \mathrm{g})$ e pulmão $(198 \mu \mathrm{g} / \mathrm{g})$, sendo as concentrações tecidulares muito superiores às séricas $(1,4)$. As doses administradas são cumulativas e têm uma semivida longa e variável (aproximadamente 100 dias) (5). Após a interrupção do tratamento, a eliminação prossegue durante meses e a persistência do efeito farmacodinâmico, durante 10 dias a um mês.

Apesar de numerosos estudos documentarem que a amiodarona exerce efeitos sobre a função tiróidea, bem como sobre o metabolismo periférico dos hormônios tiróideos, seus mecanismos de ação ainda não foram completamente elucidados. Além do excesso de iodo libertado durante seu metabolismo, que condiciona um aumento transitório nas primeiras semanas das concentrações de TSH (thyroid-stimulating hormone) secundário à supressão da síntese dos hormônios tiróideos (efeito Wolff-Chaikoff), a amiodarona também inibe a atividade das deiodinases tipo 1 e 2 . $\mathrm{O}$ retorno da TSH aos valores normais ocorre, habitualmente, após três meses e o escape a esse efeito, em condições normais, salvaguarda o desenvolvimento do HPOIA (1,4-7). Nos tecidos periféricos, especialmente em nível hepático, a amiodarona inibe a atividade da enzima $5^{\prime}$-deiodinase tipo 1 , com consequente diminuição da deiodinização periférica da $\mathrm{T}_{4}$ e r $\mathrm{T}_{3}$ ( $\mathrm{T} 3$ reverse) (5-8). Esse efeito inibitório pode persistir por mais de dois meses após suspensão da terapêutica e resulta na diminuição das concentrações séricas de $\mathrm{T}_{3}$ e aumento da $\mathrm{T}_{4}$ e $\mathrm{rT}_{3}(1,5-8)$. A inibição da atividade do 5 '-deiodinase tipo 2 , responsável pela conversão da $\mathrm{T}_{4}$ em $\mathrm{T}_{3}$ na hipófise, resulta em diminuição da $\mathrm{T}_{3}$ na hipófise e, em parte, pelo aumento inicial das concentrações séricas de TSH. Por outro lado, o fármaco afeta indiretamente o metabolismo dos hormônios tiróideos ao inibir a entrada dos hormônios nos tecidos periféricos $(7,8)$. Além da inibição enzimática descrita, a interação da amiodarona e seu principal metabólito ativo, a desetilamiodarona (DEA), pode induzir, em nível cardíaco, um estado de "bypothyroid like", ao inibir de forma competitiva e não competitiva os adre- norreceptores nucleares $\alpha$ e $\beta$ ( $\mathrm{T}_{3} \mathrm{R}-\alpha \mathrm{l}$ e $\mathrm{T}_{3} \mathrm{R}-\beta \mathrm{l}$ ) (8). Verifica-se também que o iodo em excesso tem efeito citotóxico sobre as células foliculares, levando à tiroidite destrutiva.

A incidência e a prevalência do HPOIA e HPEIA parecem estar dependentes da carência ou suficiência de iodo ambiental (9), mas variam muito entre os estudos. Em geral, estima-se que a incidência do HPEIA seja de $1 \%$ a $23 \%$, enquanto a do HPOIA seja de $1 \%$ a $32 \%(2,8,10)$. É sabido que o HPEIA é mais frequente nas áreas com déficit de iodo, enquanto o HPOIA ocorre frequentemente em áreas com suficiência de iodo no meio ambiente (1,2,5-9). Um estudo comparando o oeste da Toscânia, Itália (uma região com ingestão moderadamente baixa de iodo), e Worcester, Massachusetts (uma área com ingestão suficiente de iodo), encontrou uma frequência de HPOIA de $22 \%$ na Worcester e de $5 \%$ na Toscânia. Quanto ao HPEIA, a prevalência foi de 9,6\% e 2\% na Toscânia e Worcester, respectivamente (11). Portugal é um exemplo de áreas com insuficiência ligeira a moderada de iodo. Um estudo para avaliar a suficiência de iodo em Portugal, usando a concentração urinária de iodo (CUI), realizado em 17 maternidades portuguesas por Limbert e cols., foi publicado em 2010. O estudo demonstrou que apenas $16,8 \%$ das mulheres portuguesas tinham valores adequados de CUI (dose recomendada pela WHO para adultos é $150 \mu \mathrm{g} / \mathrm{l}$ ) e que a região central de Portugal possui uma CUI que varia entre 82,1 e $124,1 \mu \mathrm{g} / \mathrm{l}$, revelando uma insuficiência de iodo (12).

Apesar de a maioria dos doentes medicados com amiodarona permanecer em eutiroidia, alguns desenvolvem HPEIA ou HPOIA. A elevada incidência dessas complicações e o potencial perigo do agravamento da doença cardíaca são razões válidas para que se efetue monitorização periódica da função tiróidea em todos os doentes tratados com amiodarona. As diretivas da North American Society of Pacing and Electrophysiology recomendam doseamento da TSH e T4 livre antes do início do tratamento com amiodarona e, depois, a cada seis meses (13). Alguns autores recomendam, também, incluir na avaliação inicial o doseamento dos anticorpos antiperoxidase (anti-TPO) (1).

O objetivo desta revisão foi estudar os casos de DTIA ocorridos no nosso serviço, avaliando os aspectos clínicos e laboratoriais, bem como a abordagem e a evolução dos doentes em função do tratamento instituído e o prognóstico. 


\section{MATERIAIS E MÉTODOS}

No período compreendido entre janeiro de 1990 e dezembro de 2009, quinze doentes foram diagnosticados como tendo DTIA. Foi feita análise retrospectiva de revisão dos processos clínicos e cinco destes foram excluídos por dados insuficientes.

Identificaram-se os dados epidemiológicos (idade, sexo, residência), os antecedentes de doença tiróidea conhecida à data do diagnóstico da DTIA, a duração do tratamento com amiodarona, a existência de doseamentos anteriores de hormônio tiróideo ou anticorpos antitiróideo na fase pré e durante o tratamento com amiodarona, a manifestação clínica da disfunção tiróidea, os exames auxiliares de diagnósticos realizados, a terapêutica efetuada e a evolução clínica e laboratorial.

A confirmação laboratorial do diagnóstico de HPOIA e HPEIA foi feito por aumento da concentração sérica de TSH em combinação com níveis baixos de T4 livre e diminuição da TSH com elevação da T4 livre, respectivamente. Incluíram-se, nesta análise, apenas os doentes sob tratamento com amiodarona que desenvolveram DTIA, cujo diagnóstico foi estabelecido no internamento ou na consulta de Medicina Interna.

\section{RESULTADOS}

Dos dez doentes com DTIA, seis eram do gênero feminino e quatro, do masculino. A média de idade foi de 68,3 anos, variando entre 53 e 82 anos. Nove doentes eram provenientes do distrito de Coimbra e um, do distrito de Leiria (Gois). O tempo de duração de tratamento com amiodarona foi de 17,7 meses, variando entre 6 e 36 meses. Com exceção de um doente, cuja amiodarona foi-lhe prescrita por doença de Wolff-Parkinson-White, todos tinham como arritmia conhecida à data da prescrição da amiodarona a fibrilhação auricular.

Quanto aos antecedentes de doença tiróidea, apenas um tinha patologia tiróidea conhecida (BMN). A monitorização periódica da função tiróidea era feita em dois doentes. Os doseamentos incluíram TSH e T4 livre e foram solicitados após o início do tratamento com amiodarona, com presença em ambos de elevação discreta e isolada da TSH. Em nenhum doente foram doseados os anticorpos antitiróideo.

Da DTIA identificada, seis tinham HPOIA, um HPEIA tipo 1, um HPEIA tipo 3 e dois HPEIA tipo 2. O diagnóstico da DTIA foi efetuado no internamento de Medicina Interna em cinco doentes. Nestes, os motivos de internamento foram suspeita de infecção respiratória (pulmão da amiodarona?) (casos 1 e 8), insuficiência cardíaca congestiva descompensada (caso 4), febre sem foco (hepatite?) (caso 2) e suspeita de pancreatite aguda (caso 9). Nos restantes, o diagnóstico foi estabelecido na consulta de Medicina. A distribuição dos dez doentes mostra-se na tabela 1 .

A média de idade no HPOIA foi 64,83 anos e, no HPEIA, de 73,5 anos, numa amostra que variou dos 53 aos 75 anos e 66 aos 82 anos, respectivamente. Quatro dos seis doentes com HPOIA eram do sexo feminino. A distribuição por sexo foi igual no HPEIA. O tempo de duração de tratamento com amiodarona foi de 28,5 meses no HPEIA, variando entre 12 e 36 meses, e de 10,5 meses no HPOIA, variando entre 6 e 15 meses.

Verificou-se que sintomas sugestivos de HPOIA estavam presentes em quatro doentes. Destes, os sintomas predominantes foram aumento ligeiro do peso (2 doentes), obstipação (2 doentes), lentificação psicomotora ( 2 doentes) e edema da face ( 2 doentes). Os principais achados foram bradicardia ( 2 doentes), hipotermia ( 2 doentes) e hipotensão ( 1 doente). No HPEIA, a manifestação clínica mais frequente foi exacerbação da fibrilhação auricular, ocorrida em três doentes (primeiro, segundo e quarto doentes do Quadro 1).

Dos exames complementares de imagem realizados, a ecografia tiróidea realizada em oito doentes revela bócio nodular em um com HPEIA tipo 1 , alterações sugestivas de tiroidite crônica em um caso de HPOIA e tiroide heterogênea com presença de nódulos ou micronódulos em cinco doentes, dois dos quais com HPEIA. Em um doente com HPEIA tipo 2, a ecografia foi normal. A ecografia tiróidea com fluxo Doppler em cores foi realizada em dois doentes, ambos com HPEIA, revelando no HPEIA tipo 1 um aumento discreto da vascularização da glândula (grau l). Num caso de HPEIA tipo 2, o Doppler foi normal. O cintigrama tiróideo com radioisótopos marcados foi pedido em quatro doentes, três com HPEIA e um com HPOIA. Nos com HPEIA, dois com tipo 2 e um com tipo 3, a captação foi nula. No doente com HPOIA, a captação do radioisótopo foi diminuída. O doseamento de anticorpos antitiróideo foi solicitado em sete doentes, com positividade apenas em um com HPOIA. A tabela 2 apresenta uma descrição detalhada dos achados laboratoriais e imagiológicos dos dez doentes.

Em todos os doentes com HPEIA, após confirmação do diagnóstico e estabilização da taquiarritmia, optou-se pela substituição da amiodarona por outro an- 
tiarrítmico. A tionamida utilizada foi o metimazol, com doses de $15 \mathrm{mg} /$ dia nos dois primeiros doentes, de 20 $\mathrm{mg}$ no terceiro e de $30 \mathrm{mg}$ no quarto doente da tabela 1. Nos três primeiros doentes, foi instituído prednisolona, com dose inicial de $60 \mathrm{mg}$. Nos casos de HPOIA, apenas no sétimo doente do quadro 1 foi mantida a amiodarona. A levotiroxina foi instituída na dose de 0,1 mg no sexto doente e $0,05 \mathrm{mg}$ nos restantes. A descrição dos esquemas de tratamento utilizados encontra-se especificada no tabela 1 .

O tempo médio de recuperação da eutiroidia foi de 4,5 meses, sendo de 4,25 meses no HPOIA. Ao con- trário do HPEIA, verificou-se recuperação da disfunção tiróidea em todos os casos de HPOIA, contudo com necessidade de manutenção de levotiroxina nos cinco últimos doentes do tabela 1. No doente com HPEIA tipo 1, houve recorrência de descompensação cardíaca com síndrome coronária aguda após a suspensão da amiodarona e agravamento da disfunção tiróidea. Apenas no terceiro caso da tabela 1 , houve melhoria clínica e laboratorial, sendo esse mantido em eutiroidia até a presente data. Em dois doentes com HPEIA, não foi feito controle em tempo útil, dado o desfecho fatal precoce. A evolução da função tiróidea após o tratamento pode ser vista na tabela 2 .

Tabela 1. Resumo dos aspectos epidemiológicos, clínicos, tratamento e evolução da DTIA

\begin{tabular}{|c|c|c|c|c|c|c|c|c|c|c|c|}
\hline Cas. & Sx. & Id. & Resid. & Serv. & AP DT & TAM & HT & Clín. DT & Tipo DTIA & Tratam. DTIA & Evol \\
\hline 1 & $\mathrm{~F}$ & 82 & Gois & Intern. & BMN & 24 & Não & HPE & HPE 1 & $C T » A T+C T$ & Óbito \\
\hline 2 & $\mathrm{M}$ & 66 & Anadia & Intern. & - & 24 & Não & - & HPE 3 & $\mathrm{AT}+\mathrm{CT}$ & Óbito \\
\hline 3 & $\mathrm{M}$ & 68 & Coimbra & Cons. & - & 30 & Não & - & HPE 2 & $\mathrm{AT}+\mathrm{CT}$ & Melh \\
\hline 4 & $F$ & 78 & Coimbra & Intern. & - & 36 & Não & - & HPE 2 & AT & Óbito \\
\hline 5 & $\mathrm{M}$ & 53 & Pombal & Cons. & - & 12 & Não & HPO & HPO & HT & Melh \\
\hline 6 & $\mathrm{M}$ & 63 & Condeixa & Cons. & - & 8 & Não & HPO & HPO & HT & Melh* \\
\hline 7 & $\mathrm{~F}$ & 64 & Coimbra & Cons. & - & 15 & Sim & - & HPO & HT & Melh* \\
\hline 8 & $\mathrm{~F}$ & 64 & Souselas & Intern. & - & 6 & Sim & - & HPO & $\mathrm{AM}+\mathrm{HT}$ & Melh \\
\hline 9 & $\mathrm{~F}$ & 70 & Coimbra & Intern. & - & 7 & Sim & HPO & HPO & HT & Melh* \\
\hline 10 & $\mathrm{~F}$ & 75 & Coimbra & Cons. & - & 18 & Não & HPO & HPO & HT & Melh* \\
\hline
\end{tabular}

Cas.: caso clínico; Sx.: sexo; Id.: idade à data do diagnóstico da DTIA; Resid.: residência; Serv.: local onde foi feito o diagnóstico; AP DT: antecedentes de disfunção tiróidea (DT) conhecida à data do diagnóstico; TAM: duração do tratamento com amiodarona (em meses); HT: doseamento de hormônio tiróideo durante o tratamento com AM, prévio a DTIA; Clín. DT: sintomas ou sinais sugestivos de hipotiroidismo (HPO) ou hipertiroidismo (HPE); M: masculino; F: feminino; Intern.: internamento; Cons.: consulta; AT: só antiróideo; CT: só corticoide; AT + CT: antiróideo e corticoterapia; HT: só levotiroxina; AM + HT: amiodarona e levotiroxina; Melh*: melhoria, mas com necessidade de levotiroxina; (»): alteração; (-): ausência; Evol: evolução.

Tabela 2. Distribuição dos doentes quanto aos exames auxiliares de diagnóstico e resultados da função tiróidea antes e após o tratamento da DTIA

\begin{tabular}{|c|c|c|c|c|c|c|c|c|c|c|c|}
\hline \multicolumn{5}{|c|}{ Exames diagnósticos } & \multicolumn{3}{|c|}{ Antes do tratamento } & \multicolumn{3}{|c|}{ Pós-tratamento } & \multirow{2}{*}{$\begin{array}{c}\text { Eutiro } \\
\text { (M) }\end{array}$} \\
\hline Cs & Acs & Ecografia Tir & Ecod Tir & CintigraTir & $\mathrm{TSH}^{*}$ & T3I & T4I & THS $^{*}$ & T3I & T4I & \\
\hline 1 & Neg. & BMN & Grau 1 & - & 0,007 & $0,9^{1}$ & $3,7^{4}$ & 0,013 & $2^{1}$ & $5,8^{4}$ & - \\
\hline 2 & Neg. & Nódulos & - & Ausente & 0,004 & $3,7^{1}$ & $4,7^{4}$ & - & - & - & - \\
\hline 3 & Neg. & Norm. & Norm. & Ausente & 0,01 & $13,2^{1}$ & $7,77^{4}$ & 4,6 & $3,9^{1}$ & $1,1^{4}$ & 6 \\
\hline 4 & - & Heterog. & - & Ausente & 0,008 & $3,6^{1}$ & $3,4^{4}$ & - & - & - & - \\
\hline 5 & - & - & - & - & 123 & $85,3^{2}$ & $0,01^{5}$ & 3,5 & $406^{3}$ & $1,3^{5}$ & 5 \\
\hline 6 & Neg. & - & - & - & 120 & $91^{3}$ & $0,3^{5}$ & 4,5 & $437^{3}$ & $0,9^{5}$ & 6 \\
\hline 7 & Neg. & Nódulos & - & Diminui & 5,7 & $229^{3}$ & $0,5^{5}$ & 3,7 & $284^{3}$ & $1,1^{5}$ & 3 \\
\hline 8 & Neg. & Nódulos & - & - & 6,3 & $217^{3}$ & $0,6^{5}$ & 4,4 & $335^{3}$ & $1,1^{5}$ & 1,5 \\
\hline 9 & - & Ti. crônica & - & - & 61 & $50^{3}$ & Ind. & 1,9 & $368^{3}$ & $1,9^{5}$ & 2 \\
\hline 10 & Pos. & Nódulos & - & - & 10 & $3,1^{1}$ & $0,7^{4}$ & 0,9 & $3,5^{1}$ & $1,5^{4}$ & 15 \\
\hline
\end{tabular}

Eutiro.: tempo de recuperação para eutiroidia (em meses); Cas: caso clínico; Acs: anticorpos antiróideos; Ecod Tir: ecografia da tiroide com Doppler em cores; Cintigra Tir: cintigrama tiróideo com radioisótopos marcados; (M): meses; Ti. crônica: tiroidite crônica; (*): uUl/ml (valor de referência 0,4 a 4); (1): pg/ml (valor de referência 1,8 a 4,2); (2): pg \% (valor de referência 140 a 440); (3): pg/\% (valor de referência 230 a 530); (4): ng/dl (valor de referência 0,8 a 1,9).

(5): ng \% (valor de referência 0,7 a 2); Ind.: indoseável; (-): não realizado; Heterog.: heterogênea; Norm.: normal. 
Registraram-se três óbitos, todos com HPEIA. Dois dos óbitos ocorreram em menos de três semanas após o diagnóstico da DTIA, com presença de coinfecção nosocomial em um caso e coma em outro. O outro óbito, na doente com HPEIA tipo 1, ocorreu passados dois meses de internamento, em contexto de síndrome coronária aguda e falência multiórgãos.

\section{DISCUSSÃO}

Tal como mencionado, a DTIA é uma complicação relativamente comum. O HPEIA é mais frequente no sexo masculino e em idades mais jovens comparativamente ao HPOIA, que é mais prevalente nas mulheres, nos mais velhos e em doentes com história prévia de tiroidite de Hashimoto $(1,2,4,5,7,14)$. A existência de antecedente conhecido de doença de Graves ou bócio nodular (BMN) é fator de risco para o HPEIA tipo 1, cujo hipertiroidismo é induzido pela libertação de iodo da amiodarona (fenômeno de Jod-Basedow) (7). O HPEIA tipo 2, semelhante à tiroidite subaguda, ocorre em doentes sem doença tiróidea conhecida e é devido à destruição do epitélio folicular, com libertação dos hormônios tiróideos para a circulação $(1,4-8)$. Quando os tipos 1 e 2 coexistem, combinando achados de doença tiróidea preexistente (por exemplo, tiroide multinodular) com evidência de tiroidite, fala-se do HPEIA tipo 3 ou misto $(6,9)$. Por análise retrospectiva, um dos casos de HPEIA enquadra-se na classificação tipo 3.

De fato, na presente série de casos verificou-se uma maior de prevalência de HPOIA no sexo feminino e ocorrência de um caso de HPEIA tipo 1 em uma doente com BMN. Quanto à distribuição por idades, verificou-se que o HPEIA ocorreu nos mais idosos, ao contrário do que é referido na literatura.

Nesta análise, tal como está descrito na literatura, o HPOIA desenvolve-se geralmente nos primeiros 18 meses de tratamento, enquanto o HPEIA pode ocorrer quatro meses a três anos após o início do tratamento com amiodarona ou vários meses após sua suspensão e não é dependente das doses, diária ou acumulada $(1,4,7,14)$. Verificou-se na nossa série um tempo médio de desenvolvimento do HPOIA de 10,16 meses e do HPEIA de 28,5 meses, em uma amostra que variou dos seis aos 15 meses e dos 24 aos 36 meses, respectivamente.

Clinicamente, o HPEIA pode desenvolver-se de forma súbita e deve ser suspeitado se há agravamento da cardiopatia de base e exacerbação ou surgimento de taquicar- dia ou fibrilhação auricular previamente controlados pelo tratamento com amiodarona, tal como se verificou em três doentes com HPEIA da nossa série $(1,2,5,7,8)$. Deve ser considerado, ainda, em doentes que desenvolvem de forma inexplicada perda ponderal, fraqueza muscular, intolerância ao calor, tremores, palpitações ou diarreia. Raramente, o HPEIA tipo 2 pode se apresentar com dor em nível da tiroide, febre e outros sintomas sistêmicos (5). A oftalmoplegia está habitualmente ausente, exceto se ocorrer em doentes com doença de Graves $(1,7,8)$. No entanto, na maioria das vezes os doentes com HPEIA estão assintomáticos e o diagnóstico clínico torna-se bastante difícil, pois as manifestações de hipertiroidismo podem estar mascaradas pela ação antiadrenérgica da amiodarona e pelo seu efeito inibidor da conversão de T4 em T3 $(7,8)$. Este fato foi documentado nos casos aqui descritos, em que nenhum teve manifestações típicas de hipertiroidismo. Quanto ao HPOIA, a clínica é normalmente vaga e não difere das outras causas de hipotiroidismo, como se verificou nos casos apresentados. Fadiga, lentificação, intolerância ao frio e pele seca são, habitualmente, reportadas na literatura $(1,3,8)$. A ocorrência de bócio e coma mixedematoso é, excepcionalmente, rara $(1,3)$. A bradicardia foi o achado clínico encontrado em quase todos os doentes com HPOIA da série de casos apresentados.

O diagnóstico laboratorial do HPOIA é baseado na elevação sérica da TSH (exceto nos três primeiros meses, em que este efeito é normal) e diminuição do T4 livre, e do HPEIA na diminuição ou supressão da TSH, T3 livre normal ou diminuído e elevação do T4 livre $(1,2,5,7,8)$. O diagnóstico diferencial dos subtipos de HPEIA assenta na conjugação dos parâmetros clínicos (história prévia de doença tiróidea? bócio ao exame físico?), laboratoriais (anticorpos antiróideos?) e imagiológicos (cintigrama e ecografia com Doppler da tiroide). A presença de anticorpos antiróideos positivos é indicadora de doença tiróidea prévia e a favor do HPEIA tipo 1 , embora pode estar presente em cerca de $8 \%$ de casos de HPEIA tipo 2 (1). Originalmente, a interleucina-6 (IL-6) foi considerada como um grande marcador de diferenciação, tendo sido reportado que o HPEIA tipo 2 se caracterizava por um aumento expressivo dos níveis da IL-6, enquanto no tipo 1 o aumento era moderado ou ausente (7). Ultimamente, tem vindo a ser reconhecido por muito investigadores que a IL-6 não é eficaz na diferenciação $(1,5,9)$. Outros marcadores inflamatórios, como PCR e velocidade de sedimentação, 
mostraram ser ineficazes na distinção dos dois tipos de HPEIA $(1,5,9)$. O cintigrama tiróideo com radioisótopos marcados pode ser de grande valia na diferenciação dos dois tipos: no tipo 1 , a atividade aumentada do tecido tiróideo pode resultar num cintigrama normal ou com captação aumentada de Iodo 131, enquanto no tipo 2 a destruição do tecido tiróideo resulta em captação reduzida ou ausente de iodo $(1,5,6,8,9)$. Finalmente, a ecografia da tiroide com Doppler em cores, um método fácil, de rápida execução, não invasivo e barato, pode ser útil, uma vez que revela no tipo 1 um aumento da vascularização da glândula que pode variar desde grau 1 (discreto) ao grau 3 (marcado). No tipo 2 , a vascularização é de grau 0 (ausente) (9). Na prática, o diagnóstico diferencial entre os subtipos de HPEIA é complexo e pouco claro, mas muito importante, porque constitui a base racional para abordagem terapêutica. Como se verificou em dois dos casos expostos (casos 1 e 4), a distinção entre HPEIA tipo 1 e tipo 2 é crucial e suscita muitas dificuldades, com consequente escolha inadequada do tratamento a efetuar.

O tratamento do HPEIA tem sido, na maior parte das vezes, difícil, pouco eficaz e mais prolongado, o que é de se esperar se atendermos as características do fármaco. O problema que muitas vezes se coloca é a continuação ou não da terapêutica com amiodarona. Alguns autores defendem sua continuação com os seguintes argumentos: a suspensão pode não proteger a tiroide dos seus efeitos contínuos devido a sua longa semivida; a amiodarona e a DEA podem proteger o coração do excesso de hormônios tiróideos pelo efeito bypothyroid like, bem como pelo efeito betabloqueante; finalmente, $o$ benefício da suspensão pode ser inferior ao risco de recorrência da arritmia $(5,8)$. Contudo, devido ao HPEIA ser mais difícil de tratar que a tireotoxicose isolada, a opção da interrupção ou não da amiodarona deve basear-se na severidade do hipertiroidismo e, principalmente, na doença cardíaca $(1,4)$. Embora alguns autores recomendem suspender a amiodarona na HPEIA tipo $1(1,4,5)$, até a data não existem estudos clínicos randomizados que apoiem essa decisão. Um trabalho retrospectivo publicado por Osman e cols. em 2002, realizado na Inglaterra, demonstra que em doentes com HPEIA, independentemente do subtipo, a continuação da amiodarona não acarreta influência sobre o tratamento. Neste estudo e com base na continuação ou não da amiodarona, houve a discriminação de dois grupos de doentes em relação à evolução posterior. Não se verificaram, entre os dois grupos, diferença no tratamento efetuado, tempo de melhoria da função tiróidea, recaída do hipertiroidismo e ocorrência de eutiroidismo espontâneo. Segundo esse estudo, a decisão de interromper a amiodarona deve ser baseada essencialmente na condição cardíaca porque o sucesso do tratamento antitiróideo não depende da interrupção da amiodarona (15). Na nossa série, houve interrupção da amiodarona em todos os doentes com HPEIA, com recorrência da taquiarritmia apenas no caso 1 , que culminou posteriormente em síndrome coronária aguda e falência multiórgãos.

As abordagens terapêuticas dos doentes com HPEIA analisadas na série de casos transmitem a ideia de terem sido essencialmente empíricas. Verificou-se que no caso 1 , em que a doente apresentava características inequívocas de HPEIA tipo 1, a escolha inicial foi a instituição de corticoide, tendo-se, posteriormente, sido adicionadas tionamidas (dose $15 \mathrm{mg} / \mathrm{dia}$ ) associadas a corticoide. Já no caso 4, cuja análise é mais sugestiva de tipo 2, o tratamento baseou-se na administração isolada de tionamidas. Ambas as opções não são apoiadas por nenhuma referência bibliográfica.

As evidências científicas indicam que no HPEIA tipo 1 o objetivo principal é bloquear a síntese de hormônios tiróideos e a incorporação do iodo, pelo que devem ser administradas tionamidas em doses superiores às habituais ( 40 a $80 \mathrm{mg}$ de metimazol ou 600 a 800 $\mathrm{mg}$ de propiltiouracilo, por três a seis meses) devido à maior resistência, secundária à elevada concentração de iodo na glândula tiróidea $(5,7,8)$. Eventualmente, pode-se acrescentar à terapêutica perclorato de potássio (1.000 mg dia, por semanas ou meses) para inibir a entrada de iodo na tiroide naqueles doentes que não responderam às tionamidas passados dois a três meses $(1,5)$. O tratamento utilizando administração de perclorato de potássio e metimazol, de modo simultâneo, foi inicialmente reportado na literatura em 1986 por Martino e cols. Neste estudo, com uma população de 23 doentes subdivididos em três grupos (doentes tratados com $40 \mathrm{mg} /$ dia de metimazol ou com associação de metimazol e perclorato de potássio na dose $1 \mathrm{~g} /$ dia ou sem tratamento), apenas foi verificado controle da tireotoxicose no grupo associação (16). No entanto, precauções devem ser tomadas porque a frequência de efeitos adversos com tionamidas e perclorato de potássio, como a agranulocitose e anemia aplástica, é dose-dependente $(5,8)$. Está descrito que a frequência de agranulocitose em doentes tratados com perclorato de potássio é de $0,3 \%$ em 1.200 doentes, enquanto a da tionamida é de $0,94 \%$ em 10.131 doentes tratados. 
A incidência aumenta para $16 \%$ a $18 \%$ quando a dose do perclorato de potássio excede $1 \mathrm{~g} /$ dia (8). A adição de carbonato de lítio (900 a $1.350 \mathrm{mg} /$ dia, por 4 a 6 semanas) ao propiltiouracil tem sido reportada em pequenas séries com o objetivo de encurtar o tempo necessário para atingir o eutiroidismo $(1,8)$. Contudo, esses estudos requerem confirmação.

No HPEIA tipo 2, as tionamidas e o perclorato de potássio não estão indicados, pois estamos perante uma tiroidite destrutiva. Nestes, utilizam-se os corticoides (15 a $80 \mathrm{mg}$ de prednisolona ou 3 a $6 \mathrm{mg}$ de dexametasona, por 7 a 12 semanas, com redução progressiva) em decorrência de suas propriedades anti-inflamatórias $(1,8)$. No tipo 3, a combinação de metimazole, perclorato de potássio e corticoide é, provavelmente, o esquema terapêutico mais benéfico $(8)$.

Nos doentes resistentes ao tratamento médico, a tiroidectomia representa uma alternativa válida. Nessa situação, um controle rápido da tireotoxicose antes da cirurgia com ácido iopanoico pode reduzir os riscos de cirurgia em um doente com cardiopatia $(1,5)$. O tratamento com iodo radioativo geralmente não é possível, principalmente no tipo 2 , devido à captação suprimida ou baixa desse isótopo. A plasmaferese pode ser utilizada com o objetivo de eliminar o excesso de hormônios da circulação, mas seu efeito é transitório e é seguido de exacerbação do hipertiroidismo $(1,8)$.

No HPOIA, o tratamento é simples e consiste na terapêutica substitutiva com levotiroxina (normalmente em doses superiores às habitualmente utilizadas no hipotiroidismo convencional) e, sempre que possível, na continuação da amiodarona. A levotiroxina deve ser iniciada em doses de 25 a $50 \mu \mathrm{g} /$ dia e aumentada lentamente a cada 4 a 6 semanas, consoante o nível sérico da TSH $(2,5)$. Alguns autores sugerem a administração do perclorato de potássio (500 mg duas vezes/dia) durante um mês, com objetivo de encurtar o intervalo entre a interrupção da amiodarona e o eutiroidismo $(1,2,5,7,8)$. A monitorização do tratamento assenta no doseamento da TSH, que deve situar-se na metade superior do intervalo normal $(7,8)$.

Embora o tratamento da DTIA seja por vezes complexo, o eutiroidismo pode ser restaurado na maioria dos casos. O tempo médio de retorno ao eutiroidismo na nossa série foi de quatro meses no HPOIA, exceto no doente com anticorpos TPO em que o tempo foi mais prolongado, e de seis meses no HPEIA tipo II (único doente), que está em conformidade com os tempos relatados na literatura $(4,5,7)$. Deve-se salientar que a continuação da amiodarona no caso 7 não teve qualquer influência no retorno ao eutiroidismo, quando comparado nos que foi feita a suspensão.

Tal como descrito na literatura, na nossa série de casos ficou patente que o HPEIA é uma complicação potencialmente fatal. Verificou-se uma taxa de mortalidade de $75 \%$, se considerarmos apenas os doentes com HPEIA (três em quatro doentes). Tal fato poderá ser explicado por descompensação cardíaca, talvez secundária à suspensão da amiodarona mas também pela própria tireotoxicose, bem como pela escolha inadequada do tratamento devido às dúvidas na caracterização do subtipo e, ainda, pela coinfecção nosocomial.

Nossa análise de casos padece de várias limitações, entre as quais sua natureza retrospectiva e uma amostra muito reduzida.

Conclui-se que o HPEIA continua a ser uma complicação potencialmente fatal e a que causa maiores dificuldades no diagnóstico e na abordagem terapêutica, quando comparada com HPOIA. As decisões na abordagem terapêutica no HPEIA e da interrupção da amiodarona devem basear-se, se possível, na identificação do subtipo e na condição cardíaca subjacente. Os sinais e sintomas clínicos da DTIA são vagos, pelo que devem ser doseados TSH e T4 livre rotineiramente em todos os doentes sob tratamento com amiodarona.

Declaração: os autores declaram não haver conflitos de interesse científico neste estudo.

\section{REFERÊNCIAS}

1. Eskes SA, Wiersinga WM. Amiodarone and thyroid. Best Pract Res Clin Endocrinol Metab. 2009;23:735-51.

2. Harjai KJ, Licata AA. Effects of amiodarone on thyroid function. Ann Intern Med. 1997:126:63-73.

3. Kinsara AJ. Amiodarone in the new ACLS guidelines. Circulation. 2001;104:E44.

4. Wiersinga WM. Amiodarone and thyroid. Encyclopedia of Endocrine Diseases. 2004;1:192-6.

5. Basaria S, Cooper DS. Amiodarone and the thyroid. Am J Med. 2005;118:706-14.

6. Ross IL, Marshall D, Okreglicki A, Isaacs S, Levitt NS. Amiodarone-induced thyroid dysfunction. S Afr Med J. 2005;95:180-3.

7. Newman CM, Price A, Davies DW, Gray T, Weetman A. Amiodarone and the thyroid: a practical guide to the management of thyroid dysfunction induced by amiodarone therapy. Heart. 1998;79:121-7.

8. Martino E, Bartalena L, Bogazzi F, Braverman LE. The effects of amiodarone on the thyroid. Endocr Rev. 2001;22:240-54.

9. Bogazzy F, Bartalena L, Martino E. Approach to the patient with amiodarona-induced thyrotoxicosis. J Clin Endocrinol Metab. 2010;95:2529-35.

10. Trip MD, Wiersinga $W$, Plomp TA. Incidence, predictability and pathogenesis of amiodarona-induced thyrotoxicosis and hypothyroidism. Am J Med. 1991;91:507-11. 
11. Martino E, Safran M, Aghini-Lombardi F, Rajatanavin R, Lenziardi $M$, Fay $M$, et al. Environmental iodine intake and thyroid dysfunction during chronic amiodarone therapy. Ann Intern Med. 1984;101:28-34.

12. Limbert $E$, Prazeres $S$, São Pedro $M$, Madureira D, Miranda A, Ribeiro $\mathrm{M}$, et al.; Thyroid Study Group of the Portuguese Endocrine Society. lodine intake in Portuguese pregnant women: results of a countrywide study. Eur J Endocrinol. 2010;163:631-5.

13. Goldschalger N, Epstein AE, Narcarelli G, Olshansky B, Singh B. Practical guidelines for clinicians who treat patients with amioda- rone. Practice Guidelines Subcommittee, North American Society of Pacing and Electrophysiology. Arch Intern Med. 2000;160:1741-8.

14. Tavares AB, Paula SK, Vaisman M, Teixeira Pde F. Amiodarone and thyrotoxicosis: case reports. Arq Bras Cardiol. 2010;95(5):e122-4.

15. Basaria S, Cooper D. Amiodarone and the thyroid. Am J Med. 2005;118:706-14.

16. Martino E, Aghini-Lombardi F, Mariotti S, Lenziardi M, Baschieri L, Braverman LE, et al. Treatment of amiodarone associated thyrotoxicosis by simultaneous administration of potassium perchlorate and methimazole. J Endocrinol Invest. 1986;9:201-7. 